\title{
Dialysis Disequilibrium Syndrome
}

National Cancer Institute

\section{Source}

National Cancer Institute. Dialysis Disequilibrium Syndrome. NCI Thesaurus. Code C114781.

A complication occurring during hemodialysis that is thought to be due to a rapid decrease in blood urea nitrogen, and is characterized by an increase in intracranial pressure resulting in nausea, headache, vomiting, restlessness, and/or a decreased level of consciousness. 